J. Appl. Numer. Optim. 1 (2019), No. 1, pp. 63-75

Available online at http://jano.biemdas.com

https://doi.org/10.23952/jano.1.2019.1.06

\title{
KARUSH-KUHN-TUCKER OPTIMALITY CONDITIONS FOR NONSMOOTH MULTIOBJECTIVE SEMIDEFINITE AND SEMI-INFINITE PROGRAMMING
}

\author{
LE THANH TUNG
}

Department of Mathematics, College of Natural Sciences, Can Tho University, Can Tho 900000, Vietnam

\begin{abstract}
In this paper, a nonsmooth multiobjective semidefinite and semi-infinite programming is investigated. By using tangential subdifferentials for the tangential convex functions defined on the space of symmetric matrices, we establish the necessary and sufficient optimality conditions for some kind of efficient solutions of the nonsmooth multiobjective semidefinite and semi-infinite programming.

Keywords. Multiobjective semidefinite and semi-infinite programming; Tangential subdifferential; Efficient solution; Weakly efficient solution; Karush-Kunh-Tucker optimality conditions.
\end{abstract}

2010 Mathematics Subject Classification. 90C22, 90C29, 99C34, 90C46.

\section{INTRODUCTION}

Multiobjective nonlinear semidefinite programming can be viewed as an extension of multiobjective nonlinear programming, where the vector variable is replaced by symmetric semidefinite matrix. Semidefinite programming has an important role in some aspects of mathematics such as in control theory, approximation theory, combinatorial optimization and eigenvalue optimization. Various results about the applications and some algorithms of semidefinite programming can be found in $[2,7,22,23]$ and the references therein. Recently, optimality conditions in several kinds of semidefinite programming and related problems has attracted a lot of attention. Such conditions for convex semidefinite vector optimization problems were considered in [15]. In [4], first-order optimality conditions for mathematical programs with semidefinite cone complementarity constraints were established. By analyzing the manifold structure of some cones, the simplified optimality conditions in semidefinite programming were given in [17]. Sufficient conditions for global optimality of semidefinite optimization were considered in [19]. Using upper semi-regular convexificators and some generalized constraint qualifications, Fritz John type and Karush-Kuhn-Tucker type of optimality conditions of semidefinite programming were given in [6]. By using Fréchet pseudo-Jacobian and pseudo-Hessian in [8] for the vector-valued maps defined on the space of symmetric matrices, we established the first and second-order optimality conditions for some kind of efficient solutions of the vector nonsmooth semidefinite programming in [11]. The paper [25] obtains some optimality conditions for semidefinite programming via the image space analysis. On the other hand, a simultaneous minimization of a finite number of objective functions over an infinite number of constraints is called a multiobjective semi-infinite programming problem. For some recent results in

E-mail address: 1ttung@ctu.edu.vn.

Received February 20, 2019; Accepted March 22, 2019.

(C)2019 Journal of Applied and Numerical Optimization 
this direction, see, e.g., $[9,12,13,24]$ and references therein. In [14], semidefinite and semi-infinite programming problems, which includes semi-infinite linear programs and semi-definite programs as special cases, was investigated. To the best of our knowledge, there is no paper dealing with a multiobjective semidefinite and semi-infinite programming.

Inspired by these observations, we consider in this paper the optimality conditions of the multiobjective semidefinite and semi-infinite programming. The organization of this paper is as follows. In Section 2 , we recall notions needed in the sequel and propose the tangential subdifferentials for the functions defined on the space of symmetric matrices. The nonsmooth multiobjective semidefinite and semi-infinite programming is defined in the first part of Section 3. Then, the necessary and sufficient optimality conditions for some some kind of efficient solutions of the nonsmooth multiobjective semidefinite and semi-infinite programming are discussed. Some examples are also provided to illustrate our results.

\section{PRELIMINARIES}

The following notations and definitions in this section will be used throughout the paper.

Let $\mathbb{S}^{n}$ be the space of $n \times n$ symmetric matrices and let $\theta$ be zero matrix in $\mathbb{S}^{n}$. $B_{\mathbb{S}^{n}}$ stands for the closed unit ball in $\mathbb{S}^{n}$. Matrix $X \in \mathbb{S}^{n}$ is said to be positive (negative) semidefinite if $v^{T} X v \geq 0$ ( $v^{T} X v \leq 0$, respectively) for all $v \in \mathbb{R}^{n}$. Denote $\mathbb{S}_{+}^{n}\left(\mathbb{S}_{-}^{n}\right)$ the set of all positive (negative) semidefinite matrices. $\mathbb{S}_{+}^{n}\left(\mathbb{S}_{-}^{n}\right.$, resp) is a closed convex cone in $\mathbb{S}^{n}$. For $X, Y \in \mathbb{S}^{n}$, we write $X-Y \succeq \theta(X-Y \preceq \theta)$ if and only if $X-Y \in \mathbb{S}_{+}^{n}\left(X-Y \in \mathbb{S}_{-}^{n}\right.$, resp). The inner product of two symmetric matrices $X, Y \in \mathbb{S}^{n}$, with $X=\left(x_{i j}\right)_{n \times n}, Y=\left(y_{i j}\right)_{n \times n}$, is defined by

$$
\langle X, Y\rangle=X \bullet Y=\sum_{i=1}^{n} \sum_{j=1}^{n} x_{i j} y_{j i}=\operatorname{tr}(X Y),
$$

where tr stands for the trace. Since $\mathbb{S}^{n}$ and the inner product $\bullet$ form an Euclidean space, there is a natural isometry [23] identifying $\mathbb{S}^{n}$ and $\mathbb{R}^{v}$, where $v:=n(n+1) / 2$. The norm associated with the above inner product is called the Frobenius norm

$$
\|X\|=\sqrt{X \bullet X}=\sqrt{\operatorname{tr}(\mathrm{XX})}=\sqrt{\sum_{i=1}^{n} \sum_{j=1}^{n} x_{i j}^{2}} .
$$

We write $\left\langle X^{*}, X\right\rangle$ for the value of a linear functional $X^{*}$ at $X \in \mathbb{S}^{n}$. If $\left\langle X^{*}, X\right\rangle \leq 0$ for all $X^{*} \in S^{*}$, where $S^{*}$ is a subset of the dual space of $\mathbb{S}^{n}$, we write $\left\langle S^{*}, X\right\rangle \leq 0$. For $S \subseteq \mathbb{S}^{n}, \operatorname{int} S, \operatorname{cl} S, \partial S, \cos$, and cone $S$ denote its interior, closure, boundary, convex hull and the cone generated by $S$ (i.e., $\{t X \mid X \in S, t \geq 0\}$ ), resp. The convex cone containing the origin generated by $S$, denoted by $\operatorname{pos} S$, is defined as

$$
\operatorname{pos} S:=\left\{X \in \mathbb{S}^{n} \mid X=\sum_{i=1}^{k} \lambda_{i} X_{i}, X_{i} \in S, \lambda_{i} \geq 0, i=1, \ldots, k\right\} .
$$

The negative polar cone and strictly negative polar cone of $S$ are defined resp by

$$
\begin{gathered}
S^{-}:=\left\{X^{*} \in \mathbb{S}^{n} \mid\left\langle X^{*}, X\right\rangle \leq 0 \forall X \in S\right\}, \\
S^{s}:=\left\{X^{*} \in \mathbb{S}^{n} \mid\left\langle X^{*}, X\right\rangle<0 \forall X \in S \backslash\{0\}\right\} .
\end{gathered}
$$

It is easy to check that $S^{s} \subset S^{-}$and if $S^{s} \neq \emptyset$, then $\mathrm{cl} S^{s}=S^{-}$. Moreover, the bipolar theorem, see, e.g., [1], states that $S^{--}=\mathrm{cl}$ coneS. For $t>0$ and $k \in \mathbb{N}, o\left(t^{k}\right)$ designates a point in a considered space depending on $t$ such that $o\left(t^{k}\right) / t^{k} \rightarrow 0$ as $t \downarrow 0$. 
Recall that the contingent (or Bouligand) cone of $S \subseteq \mathbb{S}^{n}$ at $\bar{X} \in \operatorname{cl} S$ is

$$
T(S, \bar{x}):=\left\{X \in \mathbb{S}^{n} \mid \exists \tau_{k} \downarrow 0, \exists X_{k} \rightarrow X, \forall k \in \mathbb{N}, \bar{X}+\tau_{k} X_{k} \in S\right\} .
$$

The cone of weak feasible directions to $S$ at $\bar{X}$ is

$$
F(S, \bar{X}):=\left\{X \in \mathbb{S}^{n} \mid \exists \tau_{k} \downarrow 0, \forall k, \bar{X}+\tau_{k} X \in S\right\} .
$$

Remark 2.1. The following properties can be checked directly; see $[1,10]$ for more details.

(i) $T(S, \bar{X})$ is a closed cone. $F(S, \bar{X})$ is a cone.

(ii) If $S$ is a convex set then $T(S, \bar{X})=\operatorname{clcone}(S-\bar{X})$ and $F(S, \bar{X})=\operatorname{cone}(S-\bar{X})$.

(iii) $F(S, \bar{X}) \subset T(S, \bar{X})$.

Definition 2.1. Let $\phi: \mathbb{S}^{n} \rightarrow \mathbb{R}$ and $\bar{X}, U \in \mathbb{S}^{n}$.

(i) The directional derivative (or Dini derivative) of $\phi$ at $\bar{X}$ in direction $D$ is

$$
\phi^{\prime}(X, D)=\lim _{\tau \downarrow 0} \frac{\phi(\bar{X}+\tau D)-\phi(\bar{X})}{\tau} .
$$

For $D=\theta$, define $\phi^{\prime}(\bar{X}, \theta)=0$. We say that $\phi$ is directionally differentiable at $\bar{X}$ if its directional derivative exists in all directions $D$.

(ii) The Hadamard directional derivative of $\phi$ at $\bar{X}$ in direction $D$ is

$$
\phi^{H}\left(X, D^{\prime}\right)=\lim _{\tau \downarrow 0, D^{\prime} \rightarrow D} \frac{\phi\left(\bar{X}+\tau D^{\prime}\right)-\phi(\bar{X})}{\tau} .
$$

We say that $\phi$ is Hadamard directionally differentiable at $\bar{X}$ if its Hadamard directional derivative exists in all directions $D$.

Note that if $\phi^{H}(\bar{X}, D)$ exists, then $\phi^{\prime}(\bar{X}, D)$ also exists and they are equal. Conversely, if $\phi$ is Lipschitzian on a neighborhood $U$ of $\bar{X}$, then $\phi$ is Hadamard directionally differentiable at $\bar{X}$ in every direction $D$ in which $\phi$ is directionally differentiable.

For a nonempty set $S \subseteq \mathbb{S}^{n}$, the function $\sigma_{S}: \mathbb{S}^{n} \rightarrow \mathbb{R} \cup\{+\infty\}$, defined by

$$
\sigma_{S}(D):=\sup _{X \in S}\langle X, D\rangle \forall D \in \mathbb{S}^{n},
$$

is called the support function of $S$. Note that $\sigma_{S}$ is sublinear and lower semicontinuous, i.e., $\liminf _{D^{\prime} \rightarrow D} \sigma\left(D^{\prime}\right)=$ $\sigma(D)$ for all $D \in \mathbb{S}^{n}$. Moreover, if $S$ is bounded, then $\sigma_{S}$ is finite everywhere; see [3] for more details.

Lemma 2.1. [3] If $\sigma: \mathbb{S}^{n} \rightarrow \mathbb{R} \cup\{+\infty\}$ is a lower semicontinuous, sublinear function and everywhere finite, then there exists a nonempty compact convex set $S_{\sigma}$ such that $\sigma$ is the support function of $S_{\sigma}$, or, equivalently,

$$
S_{\sigma}=\left\{X^{*} \in \mathbb{S}^{n} \mid\left\langle X^{*}, D\right\rangle \leq \sigma(D), \forall D \in \mathbb{S}^{n}\right\} .
$$

In the line of $[16,18]$, we present the following notions.

Definition 2.2. A function $\phi: \mathbb{S}^{n} \rightarrow \mathbb{R}$ is said to be tangentially convex at $\bar{X} \in \mathbb{S}^{n}$ if for every $D \in \mathbb{S}^{n}$, $\phi^{\prime}(\bar{X}, D)$ exists, is finite and the function $\phi^{\prime}(\bar{x},):. \mathbb{S}^{n} \rightarrow \mathbb{R}$ is a convex function of $D$.

Since $\phi^{\prime}(\bar{X},$.$) is positively homogeneous, if \phi$ is tangentially convex at $\bar{X}$, then $\phi^{\prime}(\bar{X},$.$) is sublinear.$ Then, by Lemma 2.1, there exists a nonempty compact convex set of $\mathbb{S}^{n}$ such that $\phi^{\prime}(\bar{X},$.$) is the support$ function of that set. 
Definition 2.3. Let $\phi: \mathbb{S}^{n} \rightarrow \mathbb{R}$ be tangentially convex at $\bar{X} \in \mathbb{S}^{n}$. The nonempty compact convex $\partial^{T} \phi(\bar{X})$ of $\mathbb{S}^{n}$ is said to be the tangential subdifferential of $\phi$ at $\bar{X}$ if $\phi^{\prime}(\bar{X}, D)=\max _{X^{*} \in \partial^{T} \phi(\bar{X})}\left\langle X^{*}, D\right\rangle$, which is equivalent to

$$
\partial^{T} \phi(\bar{X})=\left\{X^{*} \in \mathbb{S}^{n} \mid\left\langle X^{*}, D\right\rangle \leq \phi^{\prime}(\bar{X}, D), \forall D \in \mathbb{S}^{n}\right\}
$$

Example 2.1. Let $\phi: \mathbb{S}^{2} \rightarrow \mathbb{R}$ be defined by, for $X=\left[\begin{array}{ll}x_{1} & x_{2} \\ x_{2} & x_{3}\end{array}\right]$,

$$
\phi(X)=e^{x_{1}}+4 x_{2}+x_{1} x_{3} \text {. }
$$

Then, at $\bar{X}=\left[\begin{array}{ll}0 & 0 \\ 0 & 0\end{array}\right]$, one gets $\partial^{T} \phi(\bar{X})=\left\{\left[\begin{array}{ll}1 & 2 \\ 2 & 0\end{array}\right]\right\}$.

Example 2.2. Let $\phi: \mathbb{S}^{2} \rightarrow \mathbb{R}$ be defined by, for $X=\left[\begin{array}{ll}x_{1} & x_{2} \\ x_{2} & x_{3}\end{array}\right]$,

$$
\phi(X)=\left|x_{1}\right|+2 x_{2}+\max \left\{x_{3}^{3}, x_{3}\right\} \text {. }
$$

Then, at $\bar{X}=\left[\begin{array}{ll}0 & 0 \\ 0 & 0\end{array}\right]$, one has

$$
\partial^{T} \phi(\bar{X})=\left\{\left[\begin{array}{cc}
\alpha & 1 \\
1 & \beta
\end{array}\right] \mid \alpha \in[-1,1], \beta \in[0,1]\right\}
$$

Example 2.3. Let $\phi: \mathbb{S}^{2} \rightarrow \mathbb{R}$ be defined by, for $X=\left[\begin{array}{ll}x_{1} & x_{2} \\ x_{2} & x_{3}\end{array}\right]$,

$$
\phi(X)= \begin{cases}x_{1}^{2} \sin \frac{2}{x_{1}}, & \text { if } x_{1} \neq 0 \\ 0, & \text { if } x_{1}=0\end{cases}
$$

Then, at $\bar{X}=\left[\begin{array}{ll}0 & 0 \\ 0 & 0\end{array}\right]$, we have

$$
\partial^{T} \phi(\bar{X})=\left\{\left[\begin{array}{ll}
\alpha & 0 \\
0 & 0
\end{array}\right] \mid \alpha \in[-2,2]\right\} .
$$

Definition 2.4. Let $S \subset \mathbb{S}^{n}$ be a convex set, $\phi: \mathbb{S}^{n} \rightarrow \mathbb{R}$ and $\bar{X} \in S$.

(i) $\phi$ is quasiconvex at $\bar{x}$ if

$$
\forall X \in S, \phi(X) \leq \phi(\bar{X}) \Rightarrow \phi(\lambda X+(1-\lambda) \bar{X}) \leq \phi(\bar{X}) \forall \lambda \in(0,1) .
$$

(ii) $\phi$ is Dini-convex at $\bar{X}$ if $\forall X \in S, \phi(X) \geq \phi(\bar{X})+\phi^{\prime}(\bar{X}, X-\bar{X})$.

(iii) $\phi$ is strictly Dini-convex at $\bar{X}$ if $\forall X \in S \backslash\{\bar{X}\}, \phi(X)>\phi(\bar{X})+\phi^{\prime}(\bar{X}, X-\bar{X})$.

(iv) $\phi$ is Dini-pseudoconvex at $\bar{X}$ if $\forall X \in S, \phi(X)<\phi(\bar{X}) \Rightarrow \phi^{\prime}(\bar{X}, X-\bar{X})<0$.

(v) $\phi$ is strictly Dini-pseudoconvex at $\bar{X}$ if

$$
\forall X \in S \backslash\{\bar{X}\}, \phi(X) \leq \phi(\bar{X}) \Rightarrow \phi^{\prime}(\bar{X}, X-\bar{X})<0 .
$$

(vi) $\phi$ is Dini-quasiconvex at $\bar{X}$ if $\forall X \in S, \phi(X) \leq \phi(\bar{X}) \Rightarrow \phi^{\prime}(\bar{X}, X-\bar{X}) \leq 0$. 
Remark 2.2. Let $S \subset \mathbb{S}^{n}$ be a convex set, $\phi: \mathbb{S}^{n} \rightarrow \mathbb{R}$ and $\bar{X} \in S$. Suppose that $\phi$ is tangentially convex at $\bar{X}$.

(i) If $\phi$ is Dini-convex at $\bar{X}$ and $X \in S$, then

$$
\phi(X) \geq \phi(\bar{X})+\left\langle\partial^{T} \phi(\bar{X}), X-\bar{X}\right\rangle .
$$

(ii) If $\phi$ is strictly Dini-convex at $\bar{X}$ and $X \in S \backslash\{\bar{X}\}$, then

$$
\phi(X)>\phi(\bar{X})+\left\langle\partial^{T} \phi(\bar{X}), X-\bar{X}\right\rangle .
$$

(iii) If $\phi$ is Dini-pseudoconvex at $\bar{X}$ and $X \in S, \phi(X)<\phi(\bar{X})$, then

$$
\left\langle\partial^{T} \phi(\bar{X}), x-\bar{X}\right\rangle<0 .
$$

(iv) If $\phi$ is strictly Dini-pseudoconvex at $\bar{X}$ and $X \in S \backslash\{\bar{X}\}, \phi(X) \leq \phi(\bar{X})$, then

$$
\left\langle\partial^{T} \phi(\bar{X}), X-\bar{X}\right\rangle<0 .
$$

(v) If $\phi$ is Dini-quasiconvex at $\bar{X}$ and $X \in S, \phi(X) \leq \phi(\bar{X})$, then

$$
\left\langle\partial^{T} \phi(\bar{X}), X-\bar{X}\right\rangle \leq 0 .
$$

Lemma 2.2. [3] Suppose $\left\{A_{i}: i \in I\right\}$ is a finite set of matrices in $\mathbb{S}^{n}$. For any subset $J$ of $I$, define $C_{J}:=\operatorname{pos}\left\{A_{i} \mid i \in J\right\}$.

(i) The cone $C_{I}$ is the union of those cones $C_{J}$ for which the set $\left\{A_{j} \mid j \in J\right\}$ is linearly independent. Furthermore, any such cone $C_{J}$ is closed.

(ii) If the matrix $X$ lies in $\operatorname{co}\left\{A_{i}: i \in I\right\}$, then there is a subset $J \subset I$ of size at most $1+n(n+1) / 2$ such that $X \in \operatorname{co}\left\{A_{i} \mid i \in J\right\}$. It follows that if a subset of $\mathbb{S}^{n}$ is compact, then so is its convex hull.

Since there is an isometry identifying $\mathbb{S}^{n}$ and $\mathbb{R}^{n(n+1) / 2}$, the following lemmas can be checked similarly to the results in $[5,20]$.

Lemma 2.3. Let $\left\{C_{t} \mid t \in \Gamma\right\}$ be an arbitrary collection of nonempty convex $\operatorname{set}$ in $\mathbb{S}^{n}$ and $K=\operatorname{pos}\left(\bigcup_{t \in \Gamma} C_{t}\right)$. Then, every nonzero vector of $K$ can be expressed as a non-negative linear combination of $n(n+1) / 2$ or fewer linear independent vectors, each belonging to a different $C_{t}$.

Lemma 2.4. Suppose that $S, P$ are arbitrary (possibly infinite) index sets, $A_{s}$ maps $S$ onto $\mathbb{S}^{n}$, and so do $A_{p}$. Suppose that the set

$$
\operatorname{co}\left\{A_{s}, s \in S\right\}+\operatorname{pos}\left\{A_{p}, p \in P\right\}
$$

is closed. Then the following statements are equivalent:

$$
\begin{aligned}
& I:\left\{\begin{array}{l}
\left\langle A_{s}, X\right\rangle<0, s \in S, S \neq \emptyset \\
\left\langle A_{p}, X\right\rangle \leq 0, p \in P
\end{array} \text { has no solution } X \in \mathbb{S}^{n} ;\right. \\
& \text { II : } \quad \theta \in \operatorname{co}\left\{A_{s}, s \in S\right\}+\operatorname{pos}\left\{A_{p}, p \in P\right\} \text {. }
\end{aligned}
$$

Lemma 2.5. [6] If $U \in \mathbb{S}^{n}$ and $\langle U, V\rangle \geq 0$ for all $V \in \mathbb{S}_{+}^{n}$, then $U \in \mathbb{S}_{+}^{n}$. 


\section{KKT Optimality CONDitions}

In this section, we consider the following multiobjective semidefinite and semi-infinite programming:

$$
\begin{gathered}
\mathbb{R}_{+}^{m}-\min f(X):=\left(f_{1}(X), f_{2}(X), \ldots, f_{m}(X)\right) \\
\text { s.t. } \quad g_{t}(X) \leq 0, t \in T, \\
\quad X \succeq \theta,
\end{gathered}
$$

where $f_{i}, i \in I:=\{1, \ldots, m\}, g_{t}, t \in T$ are functions from $\mathbb{S}^{n}$ to $\mathbb{R}$. The index set $T$ is an arbitrary nonempty set, not necessary finite. Denote

$$
\Omega:=\left\{X \in \mathbb{S}^{n} \mid g_{t}(X) \leq 0, t \in T, X \succeq \theta\right\}
$$

and

$$
T(\bar{X}):=\left\{t \in T \mid g_{t}(\bar{X})=0\right\} .
$$

Denote $\mathbb{R}_{+}^{|T|}$ the collection of all the functions $\lambda: T \rightarrow \mathbb{R}$ taking values $\lambda_{t}$ 's positive only at finitely many points of $T$, and equal to zero at the other points. The set of active constraint multipliers at $\bar{X} \in \Omega$ is

$$
\Lambda(\bar{X}):=\left\{\lambda \in \mathbb{R}_{+}^{|T|} \mid \lambda_{t} g_{t}(\bar{X})=0, \forall t \in T\right\} .
$$

Notice that $\lambda \in \Lambda(\bar{x})$ if there exists a finite index set $K \subset T(\bar{x})$ such that $\lambda_{t}>0$ for all $t \in K$ and $\lambda_{t}=0$ for all $t \in T \backslash K$.

Definition 3.1. (i) A matrix $\bar{X} \in \Omega$ is called a locally (Pareto) efficient solution of (P), denoted by $\bar{x} \in \mathrm{LE}(\mathrm{P})$, if there exists a neighborhood $U$ of $\bar{X}$ such that, for every $X \in U \cap \Omega$,

$$
f(X)-f(\bar{X}) \notin-\mathbb{R}_{+}^{m} \backslash\{0\} .
$$

(ii) A matrix $\bar{X} \in \Omega$ is called a local weak solution of $(\mathrm{P})$, denoted by $\bar{x} \in \operatorname{LWE}(\mathrm{P})$, if there exists a neighborhood $U$ of $\bar{X}$ such that, for every $X \in U \cap \Omega$,

$$
f(X)-f(\bar{X}) \notin-\operatorname{int} \mathbb{R}_{+}^{m} .
$$

If $U=\mathbb{S}^{n}$, the word "locally" is omitted. In this case, the weakly efficient solution sets/the weakly efficient solution sets are denoted by $\mathrm{WE}(\mathrm{P}) / \mathrm{E}(\mathrm{P})$. It is easy to check that $\mathrm{LE}(\mathrm{P}) \subset \mathrm{LWE}(\mathrm{P})$.

Definition 3.2. Let $\bar{X} \in \Omega$.

(i) We say that the Assumption (A1) holds at $\bar{X} \in \Omega$ if $f_{i}, i \in I$, is Hadamard differentiable at $\bar{X}$, the function $f_{i}^{H}(\bar{X},):. \mathbb{S}^{n} \rightarrow \mathbb{R}$ is a convex function for all $i \in I$ and $g_{t}, t \in T$, are tangentially convex at $\bar{X}$.

(ii) We say that the Assumption (A2) holds at $\bar{X} \in \Omega$ if $f_{i}, i \in I$, and $g_{t}, t \in T$, are tangentially convex at $\bar{X}$.

Lemma 3.1. Let $\bar{X} \in \operatorname{LWE}(\mathrm{P})$.

(i) If (A1) holds, then $\left(\bigcup_{i=1}^{m} \partial^{T} f_{i}(\bar{X})\right)^{s} \cap T(\Omega, \bar{X})=\emptyset$.

(ii) If (A2) holds, then $\left(\bigcup_{i=1}^{m} \partial^{T} f_{i}(\bar{X})\right)^{s} \cap F(\Omega, \bar{X})=\emptyset$.

Proof. (i) Suppose to the contrary that there exists

$$
D \in\left(\bigcup_{i=1}^{m} \partial^{T} f_{i}(\bar{X})\right)^{s} \cap T(\Omega, \bar{X}) .
$$


It follows from

$$
D \in\left(\bigcup_{i=1}^{m} \partial^{T} f_{i}(\bar{X})\right)^{s}
$$

that

$$
\left\langle X^{*}, D\right\rangle<0, \forall X^{*} \in \partial^{T} f_{i}(\bar{X}), \forall i \in I .
$$

Since the function $\phi: \partial^{T} f_{i}(\bar{X}) \subset \mathbb{S}^{n} \rightarrow \mathbb{R}$, defined by

$$
\phi\left(X^{*}\right)=\left\langle X^{*}, D\right\rangle
$$

is continuous on the compact set $\partial^{T} f_{i}(\bar{X})$, there exists a point $\bar{X}^{*} \in \partial^{T} f_{i}(\bar{X})$ such that

$$
\phi\left(\bar{X}^{*}\right)=\max _{X^{*} \in \partial^{T} f_{i}(\bar{X})}\left\langle X^{*}, D\right\rangle .
$$

This derives that

$$
f_{i}^{\prime}(\bar{X}, D)=\max _{D^{*} \in \partial^{T} f_{i}(\bar{D})}\left\langle X^{*}, D\right\rangle=\left\langle\bar{X}^{*}, D\right\rangle<0, \forall i \in I .
$$

By $D \in T(\Omega, \bar{X})$, there exist $\tau_{k} \downarrow 0$ and $D_{k} \rightarrow D$ such that $\bar{X}+\tau_{k} D_{k} \in \Omega$ for all $k$. Since $\bar{X} \in \operatorname{LWE}(\mathrm{P})$, there exists, taking subsequence if necessary, an index $i_{0} \in I$ such that

$$
f_{i_{0}}^{\prime}(\bar{X}, D)=f_{i_{0}}^{H}(\bar{X}, D)=\lim _{k \rightarrow \infty} \frac{f_{i_{0}}\left(\bar{X}+\tau_{k} D_{k}\right)-f_{i_{0}}(\bar{X})}{\tau_{k}} \geq 0,
$$

contradicting to (3.1).

(ii) Reasoning by contraposition, assume the existence of

$$
D \in\left(\bigcup_{i=1}^{m} \partial^{T} f_{i}(\bar{X})\right)^{s} \cap F(\Omega, \bar{X}) .
$$

Hence, one gets that

$$
\left\langle X^{*}, D\right\rangle<0, \quad \forall D^{*} \in \partial^{T} f_{i}(\bar{X}), \forall i \in I .
$$

Since the function $\phi: \partial^{T} f_{i}(\bar{X}) \subset \mathbb{S}^{n} \rightarrow \mathbb{R}$, defined by

$$
\phi\left(X^{*}\right)=\left\langle X^{*}, d\right\rangle
$$

is continuous on the compact set $\partial^{T} f_{i}(\bar{X})$, there exists a point $\bar{X}^{*} \in \partial^{T} f_{i}(\bar{X})$ such that

$$
\phi\left(\bar{X}^{*}\right)=\max _{X^{*} \in \partial^{T} f_{i}(\bar{X})}\left\langle X^{*}, D\right\rangle .
$$

This shows that

$$
f_{i}^{\prime}(\bar{X}, D)=\max _{X^{*} \in \partial^{T} f_{i}(\bar{X})}\left\langle X^{*}, D\right\rangle=\left\langle\bar{X}^{*}, D\right\rangle<0, \forall i \in I .
$$

By $D \in F(\Omega, \bar{X})$, there exists $\tau_{k} \downarrow 0$ such that $\bar{X}+\tau_{k} D \in \Omega$ for all $k$. Since $\bar{x} \in \operatorname{LWE}(\mathrm{P})$, there exists, taking subsequence if necessary, an index $i_{0} \in I$ such that

$$
f_{i_{0}}^{\prime}(\bar{X}, D)=\lim _{k \rightarrow \infty} \frac{f_{i_{0}}\left(\bar{X}+\tau_{k} D\right)-f_{i_{0}}(\bar{X})}{\tau_{k}} \geq 0
$$

which contradicts (3.2). 
Now, we establish some KKT necessary optimality conditions for locally weakly efficient solutions of (P) under the following constraint qualifications (CQs):

$$
\begin{aligned}
& (\mathrm{ACQ}):\left(\bigcup_{i \in I(\bar{X})} \partial^{T} g_{i}(\bar{X})\right)^{-} \cap T\left(\mathbb{S}_{+}^{n}, \bar{X}\right) \subseteq T(\Omega, \bar{X}), \\
& (\mathrm{FCQ}):\left(\bigcup_{i \in I(\bar{X})} \partial^{T} g_{i}(\bar{X})\right)^{-} \cap T\left(\mathbb{S}_{+}^{n}, \bar{X}\right) \subseteq \operatorname{cl} F(\Omega, \bar{X}) .
\end{aligned}
$$

Proposition 3.1. Let $\bar{X} \in \operatorname{LWE}(\mathrm{P})$. Suppose that (Al) holds. If (ACQ) holds at $\bar{X}$ and the set

$$
\operatorname{pos}\left(\bigcup_{t \in T(\bar{X})} \partial^{T} g_{t}(\bar{X})\right)+T\left(\mathbb{S}_{+}^{n}, \bar{X}\right)^{-}
$$

is closed, then there exist $\alpha \in \mathbb{R}_{+}^{m}$ with $\sum_{i=1}^{m} \alpha_{i}=1, \lambda \in \Lambda(\bar{X})$ and $\bar{U} \in \mathbb{S}_{+}^{n}$ with $\langle\bar{U}, \bar{X}\rangle=0$ such that

$$
\theta \in \sum_{i=1}^{m} \alpha_{i} \partial^{T} f_{i}(\bar{X})+\sum_{t \in T} \lambda_{t} \partial^{T} g_{t}(\bar{X})-\bar{U}
$$

Proof. It follows from Lemma 2.2 (i) that

$$
\left(\bigcup_{i=1}^{m} \partial^{T} f_{i}(\bar{X})\right)^{s} \cap T(\Omega, \bar{X})=\emptyset
$$

The above equation together with (ACQ) implies that

$$
\left(\bigcup_{i=1}^{m} \partial^{T} f_{i}(\bar{X})\right)^{s} \cap\left(\bigcup_{t \in T(\bar{X})} \partial^{T} g_{t}(\bar{X})\right)^{-} \cap T\left(\mathbb{S}_{+}^{n}, \bar{X}\right) \subseteq\left(\bigcup_{i=1}^{m} \partial^{T} f_{i}(\bar{x})\right)^{s} \cap T(\Omega, \bar{x})=\emptyset
$$

Hence,

$$
\begin{aligned}
& \left(\operatorname{co\bigcup } \bigcup_{i=1}^{m} \partial^{T} f_{i}(\bar{X})\right)^{s} \cap\left(\operatorname{pos} \bigcup_{t \in T(\bar{X})} \partial^{T} g_{t}(\bar{X})\right)^{-} \cap T\left(\mathbb{S}_{+}^{n}, \bar{X}\right) \\
& =\left(\bigcup_{i=1}^{m} \partial^{T} f_{i}(\bar{X})\right)^{s} \cap\left(\bigcup_{t \in T(\bar{X})} \partial^{T} g_{t}(\bar{X})\right)^{-} \cap T\left(\mathbb{S}_{+}^{n}, \bar{X}\right) \\
& =\emptyset .
\end{aligned}
$$

Now, we prove that

$$
\left(\operatorname{co\bigcup } \bigcup_{i=1}^{m} \partial^{T} f_{i}(\bar{X})\right) \cap\left(-\operatorname{pos} \bigcup_{t \in T(\bar{X})} \partial^{T} g_{t}(\bar{X})-T\left(\mathbb{S}_{+}^{n}, \bar{X}\right)^{-}\right) \neq \emptyset .
$$

Suppose to the contrary that (3.5) does not hold. Since co $\bigcup_{i=1}^{m} \partial^{T} f_{i}(\bar{X})$ is a nonempty compact convex set and

$$
-\left(\operatorname{pos} \bigcup_{t \in T(\bar{X})} \partial^{T} g_{t}(\bar{X})+T\left(\mathbb{S}_{+}^{n}, \bar{X}\right)^{-}\right)
$$


is a closed convex cone, by the strong separation theorem, there exists $X \in \mathbb{S}^{n}$ such that

$$
\left\{\begin{array}{l}
\left\langle X^{*}, X\right\rangle<0, \quad \forall X^{*} \in \operatorname{co} \bigcup_{i=1}^{m} \partial^{T} f_{i}(\bar{X}), \\
\left\langle U^{*}, X\right\rangle \geq 0, \quad \forall U^{*} \in-\operatorname{pos} \bigcup_{t \in T(\bar{X})} \partial^{T} g_{t}(\bar{X})-T\left(\mathbb{S}_{+}^{n}, \bar{X}\right)^{-} .
\end{array}\right.
$$

Since pos $\bigcup_{t \in T(\bar{X})} \partial^{T} g_{t}(\bar{X})$ and $T\left(\mathbb{S}_{+}^{n}, \bar{X}\right)^{-}$are the cones containing $\theta$, we imply the existence of $X \in \mathbb{S}^{n}$ such that

$$
\begin{cases}\left\langle X^{*}, X\right\rangle<0, & \forall X^{*} \in \operatorname{co~} \bigcup_{i=1}^{m} \partial^{T} f_{i}(\bar{X}) \\ \left\langle U^{*}, X\right\rangle \geq 0, & \forall U^{*} \in-\operatorname{pos} \bigcup_{t \in T(\bar{X})} \partial^{T} g_{t}(\bar{X}), \\ \left\langle U^{*}, X\right\rangle \geq 0, & \forall U^{*} \in-T\left(\mathbb{S}_{+}^{n}, \bar{X}\right)^{-}\end{cases}
$$

The above inequalities together with $T\left(\mathbb{S}_{+}^{n}, \bar{X}\right)^{--}=T\left(\mathbb{S}_{+}^{n}, \bar{X}\right)$ yields that

$$
X \in\left(\operatorname{co\bigcup } \bigcup_{i=1}^{m} \partial^{T} f_{i}(\bar{X})\right)^{s} \cap\left(\operatorname{pos} \bigcup_{t \in T(\bar{X})} \partial^{T} g_{t}(\bar{X})\right)^{-} \cap T\left(\mathbb{S}_{+}^{n}, \bar{X}\right),
$$

which contradicts (3.4). Therefore, (3.5) holds. It follows that

$$
\theta \in \operatorname{co} \bigcup_{i=1}^{m} \partial^{T} f_{i}(\bar{X})+\operatorname{pos} \bigcup_{t \in T(\bar{X})} \partial^{T} g_{t}(\bar{X})+T\left(\mathbb{S}_{+}^{n}, \bar{X}\right)^{-} .
$$

It follows from the above inclusion and Lemma 2.4 that there exists $\alpha \in \mathbb{R}_{+}^{m}$ with $\sum_{i=1}^{m} \alpha_{i}=1, \lambda \in \Lambda(\bar{X})$ and $-\bar{U} \in T\left(\mathbb{S}_{+}^{n}, \bar{X}\right)^{-}$such that

$$
\theta \in \sum_{i=1}^{m} \alpha_{i} \partial^{T} f_{i}(\bar{X})+\sum_{t \in T} \lambda_{t} \partial^{T} g_{t}(\bar{X})-\bar{U}
$$

It follows from $-\bar{U} \in T\left(\mathbb{S}_{+}^{n}, \bar{X}\right)^{-}$that $\langle\bar{U}, X\rangle \geq 0$ for all $V \in T\left(\mathbb{S}_{+}^{n}, \bar{X}\right)=\operatorname{clcone}\left(\mathbb{S}_{+}^{n}-\bar{X}\right)$. Therefore,

$$
\langle\bar{U}, X-\bar{X}\rangle \geq 0, \forall X \in \mathbb{S}_{+}^{n} .
$$

By choosing $X=2 \bar{X} \in \mathbb{S}_{+}^{n}$ and $X=\frac{1}{2} \bar{X} \in \mathbb{S}_{+}^{n}$, one yields that $\langle\bar{U}, \bar{X}\rangle=0$, and hence,

$$
\langle\bar{U}, X\rangle \geq 0, \forall X \in \mathbb{S}_{+}^{n}
$$

Thus, we deduce from Lemma 2.5 that $\bar{U} \in \mathbb{S}_{+}^{n}$.

Proposition 3.2. Let $\bar{X} \in \mathrm{LWE}(\mathrm{P})$. Suppose that (A2) holds. If (FCQ) holds at $\bar{X}$ and the set

$$
\operatorname{pos}\left(\bigcup_{t \in T(\bar{X})} \partial^{T} g_{t}(\bar{X})\right)+T\left(\mathbb{S}_{+}^{n}, \bar{X}\right)^{-}
$$

is closed, then there exists $\alpha \in \mathbb{R}_{+}^{m}$ with $\sum_{i=1}^{m} \alpha_{i}=1, \lambda \in \Lambda(\bar{X})$ and $\bar{U} \in \mathbb{S}_{+}^{n}$ with $\langle\bar{U}, \bar{X}\rangle=0$ such that

$$
\theta \in \sum_{i=1}^{m} \alpha_{i} \partial^{T} f_{i}(\bar{X})+\sum_{t \in T} \lambda_{t} \partial^{T} g_{t}(\bar{X})-\bar{U}
$$


Proof. It follows from Lemma 2.2 (ii) that

$$
\left(\bigcup_{i=1}^{m} \partial^{T} f_{i}(\bar{X})\right)^{s} \cap F(\Omega, \bar{X})=\emptyset .
$$

This leads that

$$
\operatorname{int}\left(\left(\bigcup_{i=1}^{m} \partial^{T} f_{i}(\bar{X})\right)^{s}\right) \cap \operatorname{cl} F(\Omega, \bar{X})=\emptyset .
$$

The above equation together with (FCQ) implies that

$$
\begin{aligned}
\left(\bigcup_{i=1}^{m} \partial^{T} f_{i}(\bar{X})\right)^{s} \cap\left(\bigcup_{t \in T(\bar{X})} \partial^{T} g_{t}(\bar{X})\right)^{-} \cap T\left(\mathbb{S}_{+}^{n}, \bar{X}\right) \subseteq\left(\bigcup_{i=1}^{m} \partial^{T} f_{i}(\bar{x})\right)^{s} \cap \operatorname{cl} F(\Omega, \bar{x}) \\
=\emptyset .
\end{aligned}
$$

The proof is continued just as in the proof of Proposition 3.1.

The following example illustrates the necessary optimality condition for weakly efficient solution of (P).

Example 3.1. Let $n=2, T=[-1,0]$ and $f: \mathbb{S}^{2} \rightarrow \mathbb{R}^{2}$ and $g_{t}: \mathbb{S}^{2} \rightarrow \mathbb{R}$ be defined as

$$
\begin{gathered}
f_{1}(X)=\left|x_{1}\right|, f_{2}(X)=x_{3}, \\
g_{t}(X)=-2 x_{2}+t .
\end{gathered}
$$

Then,

$$
\Omega=\left\{X \in \mathbb{S}^{n} \mid x_{2} \geq 0, X \succeq \theta\right\}
$$

It is easy to see that

$$
\bar{X}=\left[\begin{array}{ll}
0 & 0 \\
0 & 0
\end{array}\right]
$$

is a weakly efficient solution of $(\mathrm{P})$. By some calculations, we have

$$
\begin{gathered}
\partial^{T} f_{1}(\bar{X})=\left\{\left[\begin{array}{ll}
\alpha & 0 \\
0 & 0
\end{array}\right] \mid \alpha \in[-1,1]\right\}, \partial^{T} f_{2}(\bar{X})=\left\{\left[\begin{array}{ll}
0 & 0 \\
0 & 1
\end{array}\right]\right\} \\
T(\bar{X})=\{0\}, \bigcup_{t \in T(\bar{X})} \partial^{T} g_{t}(\bar{X})=\left\{\left[\begin{array}{cc}
0 & -1 \\
-1 & 0
\end{array}\right]\right\} \\
T\left(\mathbb{S}_{+}^{n}, \bar{X}\right)=\mathbb{S}_{+}^{n}, T(\Omega, \bar{X})=\Omega .
\end{gathered}
$$

Hence,

$$
\left(\bigcup_{i \in I(\bar{X})} \partial^{T} g_{i}(\bar{X})\right)^{-} \cap T\left(\mathbb{S}_{+}^{n}, \bar{X}\right)=\left\{X \in \mathbb{S}^{n} \mid-2 x_{2} \leq 0\right\} \cap \mathbb{S}_{+}^{n} \subseteq T(\Omega, \bar{X}),
$$

i.e., (ACQ) holds at $\bar{X}$. Moreover,

$$
\operatorname{pos}\left(\bigcup_{t \in T(\bar{X})} \partial^{T} g_{t}(\bar{X})\right)+T\left(\mathbb{S}_{+}^{n}, \bar{X}\right)^{-}=\left\{\left[\begin{array}{cc}
0 & \alpha \\
\alpha & 0
\end{array}\right] \mid \alpha \leq 0\right\}-\mathbb{S}_{+}^{n}
$$


is closed, since it is the sum of two closed and finite-dimensional subspaces of $\mathbb{S}^{n}$; see, e.g., [21, Theorem 1.42]. Hence, all the assumptions of Proposition 3.1 hold. If we take

$$
\bar{U}=\left[\begin{array}{ll}
1 / 2 & 1 / 4 \\
1 / 4 & 1 / 2
\end{array}\right],
$$

$\alpha_{1}=\alpha_{2}=1 / 2, \lambda: T \rightarrow \mathbb{R}$ defined by

$$
\lambda(t)=\left\{\begin{array}{cc}
1 / 4, & \text { if } t=0 \\
0, & \text { otherwise }
\end{array}\right.
$$

then $\bar{U} \in \mathbb{S}_{+}^{2}$ with $\langle\bar{U}, \bar{X}\rangle=0$ and

$$
\theta \in \alpha_{1} \partial^{T} f_{i}(\bar{X})+\alpha_{2} \partial^{T} f_{2}(\bar{X})+\sum_{t \in T} \lambda_{t} \partial^{T} g_{t}(\bar{X})-\bar{U} .
$$

Proposition 3.3. Let $\bar{X} \in \Omega$. Suppose that there exist $\alpha \in \mathbb{R}_{+}^{m}$ with $\sum_{i=1}^{m} \alpha_{i}=1, \lambda \in \Lambda(\bar{X})$ and $\bar{U} \in \mathbb{S}_{+}^{n}$ with $\langle\bar{U}, \bar{X}\rangle=0$ such that

$$
\theta \in \sum_{i=1}^{m} \alpha_{i} \partial^{T} f_{i}(\bar{X})+\sum_{t \in T} \lambda_{t} \partial^{T} g_{t}(\bar{X})-\bar{U}
$$

(i) If $f_{i}, i \in I$, is Dini-pseudoconvex at $\bar{X}$ and $g_{t}, t \in T$, is Dini-quasiconvex $\bar{X}$, then $\bar{X}$ is a weakly efficient solution of $(\mathrm{P})$.

(ii) If $f_{i}, i \in I$, is strictly Dini-pseudoconvex at $\bar{X}$ and $g_{t}, t \in T$, is Dini-quasiconvex $\bar{X}$, then $\bar{X}$ is an efficient solution of $(\mathrm{P})$.

Proof. (i) Suppose on the contrary that $\bar{X}$ is not a weakly efficient solution. Then there exists a feasible point $X$ such that

$$
f_{i}(X)<f_{i}(\bar{X}), \quad \forall i=1, \ldots, m .
$$

Since $\bar{X} \in \Omega$ satisfies (3.16), there exist

$$
X_{i}^{*} \in \partial^{T} f_{i}(\bar{X}), i \in I, \quad Y_{t}^{*} \in \partial^{T} g_{t}(\bar{X}), t \in J,
$$

where $J$ is a finite subset of $T(\bar{X})$, and $\bar{U} \in \mathbb{S}_{+}^{n}$ such that

$$
-\sum_{t \in J} Y_{t}^{*}=\sum_{i=1}^{m} X_{t}^{*}-\bar{U}
$$

Since $f_{i}$ is Dini-pseudoconvex and $f_{i}(X)<f_{i}(\bar{X})$ for each $i$, one has

$$
\left\langle X_{i}^{*}, X-\bar{X}\right\rangle<0, \forall X_{i}^{*} \in \partial^{T} f_{i}(\bar{X}), \forall i \in I .
$$

Moreover, since $\bar{U}, X, \bar{X} \in \mathbb{S}_{+}^{n}$ and $\langle\bar{U}, \bar{X}\rangle=0$, we have

$$
\langle\bar{U}, X-\bar{X}\rangle=\langle\bar{U}, X\rangle \geq 0 .
$$

Hence, we derive from $\alpha \in \mathbb{R}_{+}^{m}$ with $\sum_{i=1}^{m} \alpha_{i}=1$, (3.9) and (3.10) that

$$
-\sum_{t \in J}\left\langle Y_{t}^{*}, X-\bar{X}\right\rangle=\sum_{i=1}^{m}\left\langle X_{t}^{*}, X-\bar{X}\right\rangle-\langle\bar{U}, X-\bar{X}\rangle<0 .
$$

On the other hand, for each $t \in J, g_{t}(X) \leq g_{t}(\bar{X})$. Therefore, by the Dini-quasiconvexity of $g_{t}, t \in T$, at $\bar{X}$, one has

$$
\sum_{t \in J}\left\langle Y_{t}^{*}, X-\bar{X}\right\rangle \leq 0
$$


or equivalently,

$$
-\sum_{t \in J}\left\langle Y_{t}^{*}, X-\bar{X}\right\rangle \geq 0
$$

contradicting with (3.11).

(ii) Reasoning by contraposition, suppose the existence of a feasible matrix $X$ and at least $i_{0} \in I$ such that

$$
\left\{\begin{array}{l}
f_{i}(X) \leq f_{i}(\bar{X}), \quad \forall i \in I \backslash\left\{i_{0}\right\}, \\
f_{i_{0}}(X)<f_{i_{0}}(\bar{X})
\end{array}\right.
$$

and hence, $X \neq \bar{X}$. Since $\bar{X} \in \Omega$ satisfies (3.16), there exist

$$
X_{i}^{*} \in \partial^{T} f_{i}(\bar{X}), i \in I, \quad Y_{t}^{*} \in \partial^{T} g_{t}(\bar{X}), t \in J
$$

where $J$ is a finite subset of $T(\bar{X})$, and $\bar{U} \in \mathbb{S}_{+}^{n}$ such that

$$
-\sum_{t \in J} Y_{t}^{*}=\sum_{i=1}^{m} X_{t}^{*}-\bar{U}
$$

It follows from the fact that $f_{i}, i \in I$, is strictly Dini-pseudoconvex, $X \neq \bar{X}$ and (3.12), one has

$$
\left\langle X_{i}^{*}, X-\bar{X}\right\rangle<0, \forall X_{i}^{*} \in \partial^{T} f_{i}(\bar{X}), \forall i \in I .
$$

Moreover, since $\bar{U}, X, \bar{X} \in \mathbb{S}_{+}^{n}$ and $\langle\bar{U}, \bar{X}\rangle=0$, we have

$$
\langle\bar{U}, X-\bar{X}\rangle=\langle\bar{U}, X\rangle \geq 0 \text {. }
$$

Hence, we derive from $\alpha \in \mathbb{R}_{+}^{m}$ with $\sum_{i=1}^{m} \alpha_{i}=1$, (3.13) and (3.14) that

$$
-\sum_{t \in J}\left\langle Y_{t}^{*}, X-\bar{X}\right\rangle=\sum_{i=1}^{m}\left\langle X_{t}^{*}, X-\bar{X}\right\rangle-\langle\bar{U}, X-\bar{X}\rangle<0
$$

On the other hand, for each $t \in J, g_{t}(X) \leq g_{t}(\bar{X})$. Therefore, by the Dini-quasiconvexity of $g_{t}, t \in T$, at $\bar{X}$, one has

$$
-\sum_{t \in J}\left\langle Y_{t}^{*}, X-\bar{X}\right\rangle \geq 0
$$

which contradicts (3.15).

Corollary 3.1. Let $\bar{X} \in \Omega$. Suppose that there exist $\alpha \in \mathbb{R}_{+}^{m}$ with $\sum_{i=1}^{m} \alpha_{i}=1, \lambda \in \Lambda(\bar{X})$ and $\bar{U} \in \mathbb{S}_{+}^{n}$ with $\langle\bar{U}, \bar{X}\rangle=0$ such that

$$
\theta \in \sum_{i=1}^{m} \alpha_{i} \partial^{T} f_{i}(\bar{X})+\sum_{t \in T} \lambda_{t} \partial^{T} g_{t}(\bar{X})-\bar{U} .
$$

(i) If $f_{i}, i \in I$ and $g_{t}, t \in T$, is Dini-convex $\bar{X}$, then $\bar{X}$ is a weakly efficient solution of (P).

(ii) If $f_{i}, i \in I$, is strictly Dini-convex at $\bar{X}$ and $g_{t}, t \in T$, is Dini-convex $\bar{X}$, then $\bar{X}$ is an efficient solution of $(\mathrm{P})$. 


\section{REFERENCES}

[1] J.P. Aubin, H. Frankowska, Set-Valued Analysis, Birkhäuser, Boston, 1990.

[2] J. F. Bonnans, A. Shapiro, Perturbation Analysis of Optimization Problems, Springer, New York, 2000.

[3] J. Borwein, A. S. Lewis, Convex Analysis and Nonlinear Optimization: Theory and Examples, Springer, New York, 2010.

[4] C. Ding, D. Sun, J.J. Ye, First order optimality conditions for mathematical programs with semidefinite cone complementarity constraints, Math. Program. 147 (2014), 539-579.

[5] M.A. Goberna and M.A. López, Linear Semi-Infinite Optimization, Wiley, Chichester, 1998.

[6] M. Golestani, S. Nobakhtian, Optimality conditions for nonsmooth semidefinite programming via convexificators, Positivity 17 (2013), 711-732.

[7] J.B. Hiriart-Urruty, J. Malick, A fresh variational-analysis look at the positive semidefinite matrices world, J. Optim. Theory Appl. 153 (2012), 551-577.

[8] V. Jeyakumar, D.T. Luc, Approximate Jacobian matrices for nonsmooth continuous maps and $C^{1}$-optimization, SIAM J. Control Optim. 36 (1998), $1815-1832$.

[9] A. Kabgani, M. Soleimani-damaneh, Characterization of (weakly/properly/robust) efficient solutions in nonsmooth semiinfinite multiobjective optimization using convexificators, Optimization 67 (2017), 217-235.

[10] A.A. Khan, C. Tammer, C. Zanilescu, Set-Valued Optimization, Springer-Verlag, Berlin, 2016.

[11] P.Q. Khanh, L.T. Tung, First- and second-order optimality conditions for vector nonsmooth semidefinite programming, 14th Workshop on Optimization and Scientific Computing, Bavi, Hanoi, Vietnam, 2016.

[12] N. Kanzi, S. Nobakhtian, Optimality conditions for nonsmooth semi-infinite multiobjective programming, Optim. Lett. 8 (2014), 1517-1528.

[13] N. Kanzi, On strong KKT optimality conditions for multiobjective semi-infinite programming problems with Lipschitzian data, Optim. Lett. 9 (2015), 1121-1129.

[14] S.J. Li, X.Q. Yang, K.L. Teo, Duality for semi-definite and semi-infinite programming, Optimization 52 (2003), $507-528$.

[15] G.M. Lee, K.B. Lee, Optimality theorems for convex semidefinite vector optimization problems, Nonlinear Anal. 71 (2009), 2540-2550.

[16] J.E. Martínez-Legaz, Optimality conditions for pseudo-convex minimization over convex sets defined by tangentially convex constraints, Optim. Lett. 9 (2015) 1017-1023.

[17] J.P. Penot, Optimality conditions in semidefinite programming, Num. Funct. Anal. Optim. 35 (2014), 1174-1196.

[18] B.N. Pshenichnyi, Necessary Conditions for an Extremum. Marcel Dekker Inc, New York, 1971.

[19] J. Quan, J. Wu, G. Li, O. Wu, Sufficient conditions for global optimality of semidefinite optimization, J. Inequal. Appl. 2012 (2012), Article ID 108.

[20] R.T. Rockafellar, Convex Analysis. Princeton Math. Ser. Vol. 28, Princeton University Press, Princeton, New Jersey, 1970.

[21] W. Rudin, Functional Analysis, McGraw-Hill Inc, New York, 1991.

[22] D. Sun, The strong second-order sufficient condition and constraint nondegeneracy in nonlinear semidefinite programming and their implications, Math. Oper. Res. 31 (2006), 761-776.

[23] D. Sun, J. Sun, Semismooth matrix-valued functions. Math. Oper. Res. 27 (2002), 150-169.

[24] L.T. Tung, Strong Karush-Kuhn-Tucker optimality conditions for multiobjective semi-infinite programming via tangential subdifferential, RAIRO Oper. Res. 52 (2018), 1019-1041.

[25] S. Yang, Semidefinite programming via image space analysis, J. Ind. Manag. Optim. 12 (2016), 1187-1197. 